\title{
Thymectomy in Pemphigus Foliaceus: A Thirty-Year Observation
}

\author{
Ralph W. Kuncla, b, c
}

\begin{abstract}
Myasthenia gravis is an archetypal human autoimmune disease. Thymectomy is proven effective by controlled clinical trials, and is commonly part of the immunotherapeutic approach when myasthenia creates generalized weakness. Pemphigus foliaceus is also autoimmune but treated medically; and thymectomy is not part of therapy unless thymoma is discovered. Autoimmune mechanisms, age distribution, and response to therapy in autoimmune disorders are likely to be different with thymoma. The concurrence of generalized myasthenia with disfiguring pemphigus foliaceus in one young patient but without thymoma offered a natural experiment to assess immunologic antibody responsiveness postoperatively, and observe more than a quarter of a century of clinical remission of both following total thymectomy surgery.
\end{abstract}

Keywords: Myasthenia gravis; Pemphigus; Thymectomy; Autoimmune disorders; Immunotherapy

\section{Introduction}

The benefit of thymectomy in generalized myasthenia gravis has been considered since arguably the first surgery for thymoma in that disease in 1936 [1], and then later for non-thymomatous myasthenia at the Johns Hopkins Hospital by Alfred Blalock, as published in 1941 - 1947 [2]. The therapeutic efficacy of thymectomy for generalized myasthenia gravis without thymoma was finally shown in a multicenter, single-blind, randomized trial published in 2016 and carried out between 2006 and 2012 [3], and its effect persists at least 5 years [4]. It is part of the standard practice of immunotherapy in generalized myasthenia, but its role in other autoimmune disorders is largely uncharted.

Manuscript submitted September 15, 2020, accepted October 22, 2020

Published online December 30, 2020

aDepartment of Neurology, The Johns Hopkins University School of Medicine, Baltimore, MD, USA

bepartment of Biology, University of Redlands, Redlands, CA, USA

${ }^{c}$ Corresponding Author: Ralph W. Kuncl, University of Redlands, 1200 E.

Colton Ave., Redlands, CA 92373, USA. Email: ralph_kunc1@redlands.edu

doi: https://doi.org/10.14740/jmc3585
The opportunity arose to evaluate the course of poorly controlled, disfiguring pemphigus foliaceus in a patient following her thymectomy for myasthenia gravis. Both diseases are antibody-mediated, and are passively transmissible transplacentally or by immunoglobulin injections of experimental animals. In myasthenia gravis, the target antigen for autoimmunity is the acetylcholine receptor (AChR), causing weakness and fatigability; and in pemphigus foliaceus it is desmoglein 1, causing loss of adhesion among keratinocytes in the superficial epidermis. Elective thymectomy for generalized myasthenia gravis may affect the afferent arc of the immune response by removing AChR-bearing myogenic cells, which offer a source of molecular mimicry, but it likely also changes autoreactive T-cell balance by removing other controllers of the efferent immune response by regulatory and helper $\mathrm{T}$ cells [5]. How thymectomy might work in pemphigus foliaceus, or even if it does, is unknown, but speculation would likely involve similar mechanisms.

The concurrence of the two autoimmune disorders is not new, and many individual case reports came out in the 1970s. Subsequently, single case reports of thymectomy in myasthenia gravis and pemphigus together were in the complicating context of thymoma, when surgery is considered nearly mandated rather than elective $[6,7]$. This is a report of therapeutic total thymectomy surgery in a case not complicated by thymoma, allowing for both assessment of disease-related antibody titers following surgery and clinical remission over more than a quarter of a century.

\section{Case Report}

The patient was 16 years old when she developed periodic disfiguring blistering lesions of the face and arms that left scars. She graded disease activity subjectively as " 8 out of 10 ," being the worst during the first $1-3$ years. The diagnosis of pemphigus foliaceus was made by physical examination and confirmed by indirect immunofluorescence of serum. The flaring attacks were affected only modestly by dapsone, so her dermatologist added oral prednisone between 5 and $10 \mathrm{mg}$ per day for better control.

As a 19-year-old second-year college student, when her pemphigus activity was "at its worst," she presented with myasthenia gravis because of problematic slurred speech and fatigability and weakness of leg muscles. Diagnosis was confirmed by repetitive nerve stimulation and by greatly elevated serum anti-AChR antibody. Imaging of the thymus did not 


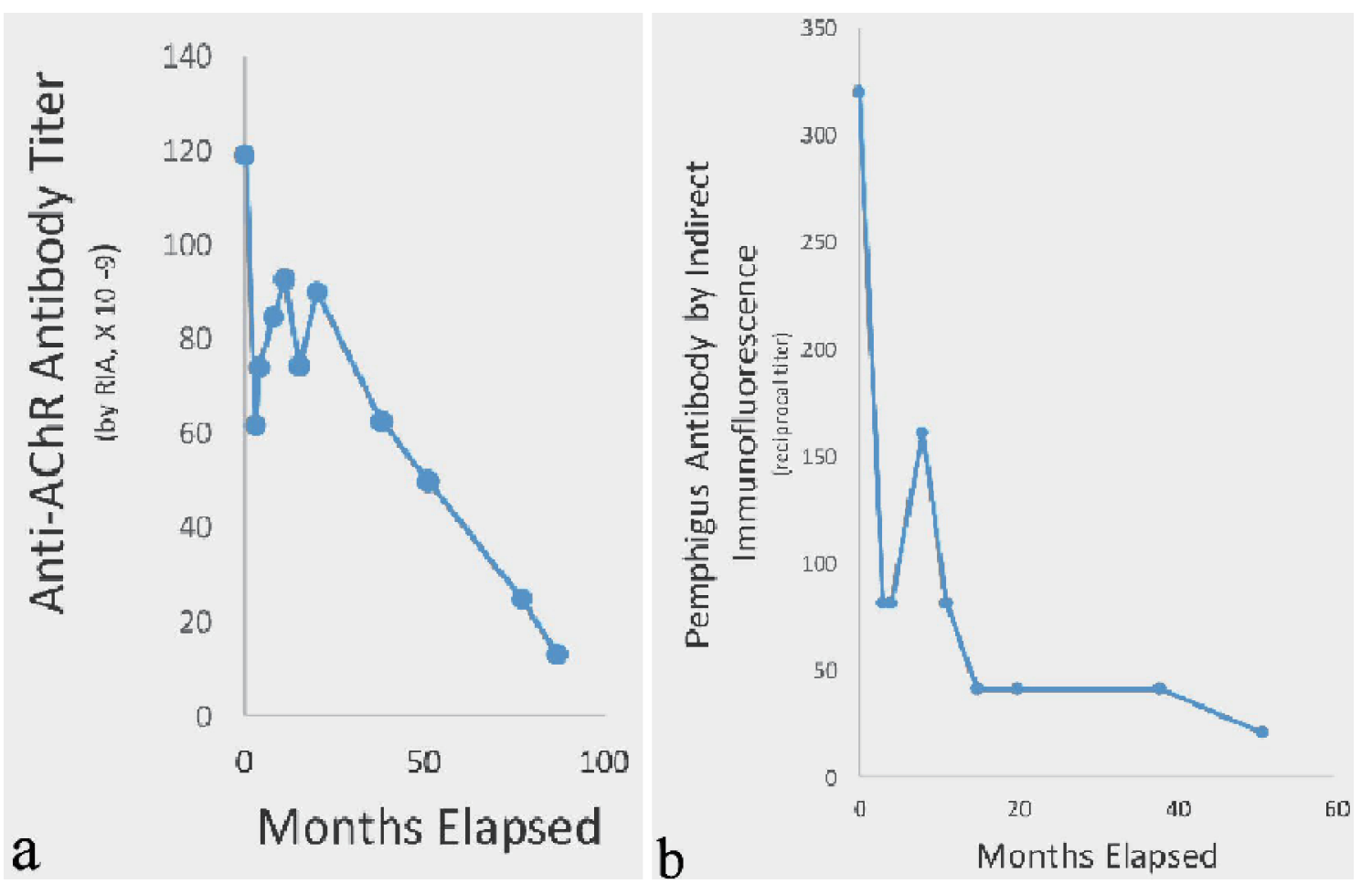

Figure 1. Response of anti-AChR antibody (a) and pemphigus antibody (b) over time before and after thymectomy. First data points at time zero indicate pre-thymectomy antibody levels 1 month before surgery. Assays for each antibody were done in one batch to reduce the errors of repeated measurements. Anti-AChR antibody titer decreased 9-fold and pemphigus antibody 16fold in parallel over time. AChR: acetylcholine receptor; RIA: radioimmunoassay.

show thymoma. She responded partially to oral pyridostigmine. The low-dose daily prednisone was not changed. She had no other autoimmune disorders.

At age 20, because of generalized myasthenia gravis, she underwent extended thymectomy by median sternotomy, with complete resection of mediastinal fat and exploration into the neck for ectopic thymic tissue. Preoperative anti-AChR antibody level by radioimmunoassay (RIA) was greatly elevated at $119 \times 10^{-9} \mathrm{M}$ (normal controls, $<0.16 \times 10^{-9} \mathrm{M}$ ). Anti-pemphigus antibody by indirect immunofluorescence was positive at 1:320 dilution (normal, undetectable).

Serum was collected periodically for diagnostic purposes (before thymectomy and for 7 years thereafter), and an aliquot was stored frozen at $-70{ }^{\circ} \mathrm{C}$ for later assay. Anti-AChR antibody was assayed by RIA using human AChR as antigen, and pemphigus antibody was assayed (by an older technique in the 1990s) by indirect immunofluorescence using squamous cell epithelium of monkey esophagus. For the construction of Figure 1, all assays for each antibody were carried out simultaneously in single batches in order to lessen the between-test variance due to conditions of measurement.

For the 6 years after thymectomy, no change was made in her low-dose oral prednisone and dapsone; only the pyridostigmine (the only drug used for her myasthenia) was gradually decreased, and the topical medications for pemphigus were altered if there were flares. By 7 months after surgery, her "skin had improved markedly," according to her dermatologist. The only short-lived rise in anti-pemphigus antibody months after thymectomy was temporally related to a brief flare in pemphigus symptoms after skin tanning exposure. Disease activity waned over time, and both lesions and flare activity became more manageable, in that there were fewer lesions on the face and much less discomfort. She needed to see her dermatologist only twice per year instead of the previous $4-8$ visits. By 6 years after surgery, prednisone for pemphigus was tapered down to $10 / 0 \mathrm{mg}$ on alternate days. By 10 years, prednisone was no longer used for pemphigus. By 16 years after surgery, when she had scant symptoms of pemphigus, she delivered a child who had transient neonatal pemphigus lasting for 1 week (but was normal for the next 13 years of life), indicating our patient still had circulating pemphigus antibody. Under vigilant surveillance for neonatal myasthenia, none developed. Likewise, the patient's symptoms of myasthenia had gradually waned after thymectomy, so that even low-dose pyridostigmine was discontinued at 10 years as she became, and remains, asymptomatic, no longer requiring neurological care.

\section{Discussion}

It is already known that myasthenia gravis and pemphigus foliaceus only rarely overlap as autoimmune disorders; that thymectomy is a proven treatment for myasthenia gravis; and that thymectomy is not performed in pemphigus unless thymoma is present.

This natural experiment of a single patient with both sys- 
temic myasthenia gravis and disfiguring pemphigus foliaceus but without thymoma shows responsiveness to thymectomy, both immunologically and by remission over a 30-year follow-up.

The 9-fold decline in anti-AChR antibody correlated with a decline in, then elimination of the need for pyridostigmine. It corresponded to a 16 -fold fall in the pemphigus antibody and substantial improvement in control of blistering and disfiguration by scarring. Remission of myasthenia has been complete; remission from pemphigus has been dramatic but by comparison not quite complete. Long-term remission of recalcitrant pemphigus vulgaris has also been shown lasting up to 10 years following an intensive course of 6 months of intravenous (IV) rituximab and IV immune globulin [8].

There are obvious limitations in this case report. This is an "N-of-one" inadvertent experiment of clinical practice, with no sham control, with open observation of the status of the disease, and with no ability to measure the relevant prethymectomy antibody trends of pemphigus or myasthenia except once. The inter-assay variability for normal controls for anti-AChR antibody by RIA is approximately $+/-0.1 \times 10^{-9} \mathrm{M}$, and for pemphigus antibody by indirect immunofluorescence is +/- 1 - 2 dilutions. Therefore, an essentially monotonic decline in both antibody titers of such magnitude implies benefit of thymectomy in both diseases, because no other systemic immunotherapy was altered during the 7 years of observation during the antibody time-trends illustrated above. Substantial falls in anti-AChR antibody titers after thymectomy predict clinical improvement in myasthenia [9]. Further, over more than a quarter century of follow-up, both disorders dramatically improved or remitted.

Perhaps thymectomy could play a role in difficult-to-treat pemphigus foliaceus, and it might even be extended to other difficult-to-treat autoimmune disorders. Now that extended thymectomy by robotic-controlled transcervical/subxiphoid videothoracoscopic surgery offers a less invasive (yet still "maximal" in dissection and effective) alternative than sternotomy [10], thymectomy might be preferable in some cases to the expense and toxicity of long-term, even lifelong immunotherapy [11].

\section{Acknowledgments}

Grant J. Anhalt MD was responsible for pemphigus immunofluorescence assays, Robert N. Adams and Daniel B. Drachman MD for anti-AChR antibody radioimmunoassay, and Gregory Bulkley MD for thymectomy surgery. All are acknowledged with thanks.

\section{Financial Disclosure}

None to declare.

\section{Conflict of Interest}

None to declare.

\section{Informed Consent}

Informed consent was obtained.

\section{Author Contributions}

RWK was solely responsible for conception, data collection, and writing.

\section{Data Availability}

The data supporting the findings of this study are available from the corresponding author upon reasonable request.

\section{References}

1. Blalock A, Mason MF, Morgan HJ, Riven SS. Myasthenia gravis and tumors of the thymic region: report of a case in which the tumor was removed. Ann Surg. 1939;110(4):544-561.

2. Kirschner PA. Alfred Blalock and thymectomy for myasthenia gravis. Ann Thorac Surg. 1987;43(3):348349.

3. Wolfe GI, Kaminski HJ, Aban IB, Minisman G, Kuo HC, Marx A, Strobel P, et al. Randomized Trial of Thymectomy in Myasthenia Gravis. N Engl J Med. 2016;375(6):511522.

4. Wolfe GI, Kaminski HJ, Aban IB, Minisman G, Kuo HC, Marx A, Strobel P, et al. Long-term effect of thymectomy plus prednisone versus prednisone alone in patients with non-thymomatous myasthenia gravis: 2-year extension of the MGTX randomised trial. Lancet Neurol. 2019;18(3):259-268.

5. Le Panse R, Berrih-Aknin S. Immunopathogenesis of myasthenia gravis. In: Kaminsky HJ, Kusner LL, eds. Myasthenia Gravis and Related Disorders, 3rd edn. Humana Press Cham (Springer). 2018; p. 47-60.

6. Takeshita K, Amano M, Shimizu T, Oyamada Y, Abiko T, Kobayashi K, Futei Y, et al. Thymoma with pemphigus foliaceus. Intern Med. 2000;39(9):742-747.

7. Yoshida M, Miyoshi T, Sakiyama S, Kondo K, Tangoku A. Pemphigus with thymoma improved by thymectomy: report of a case. Surg Today. 2013;43(7):806-808.

8. Ahmed AR, Kaveri S, Spigelman Z. Long-Term Remissions in Recalcitrant Pemphigus Vulgaris. N Engl J Med. 2015;373(27):2693-2694.

9. Kim H, Lim YM, Lee EJ, Oh YJ, Kim KK. Factors predicting remission in thymectomized patients with acetylcholine receptor antibody-positive myasthenia gravis. Muscle Nerve. 2018;58(6):796-800.

10. Zielinski M, Hauer L, Hauer J, Pankowski J, Nabialek T, Szlubowski A. Comparison of complete remission rates after 5 year follow-up of three different techniques of thymectomy for myasthenia gravis. Eur J Cardiothorac Surg. 2010;37(5):1137-1143. 
11. Gronseth GS, Barohn R, Narayanaswami P. Practice advisory: Thymectomy for myasthenia gravis (practice parameter update): Report of the Guideline Develop- ment, Dissemination, and Implementation Subcommittee of the American Academy of Neurology. Neurology. 2020;94(16):705-709. 